\title{
BMJ Open Integrated strategies to prevent intradialytic hypotension: research protocol of the DialHypot study, a prospective randomised clinical trial in hypotension-prone haemodialysis patients
}

\author{
Francesco Peyronel (DD , ${ }^{1,2}$ Elisabetta Parenti, ${ }^{1}$ Paride Fenaroli, ${ }^{1,2}$ \\ Giuseppe Daniele Benigno, ${ }^{1,2}$ Giovanni Maria Rossi, ${ }^{1}$ Umberto Maggiore, ${ }^{1,2}$ \\ Enrico Fiaccadori ${ }^{1,2}$
}

To cite: Peyronel F, Parenti E, Fenaroli P, et al. Integrated strategies to prevent intradialytic hypotension: research protocol of the Diallypot study, a prospective randomised clinical trial in hypotensionprone haemodialysis patients. BMJ Open 2020;10:e036893. doi:10.1136/ bmjopen-2020-036893

- Prepublication history and additional material for this paper are available online. To view these files, please visit the journal online (http://dx.doi org/10.1136/bmjopen-2020036893).

Received 10 January 2020 Revised 03 June 2020 Accepted 04 June 2020

Check for updates

(C) Author(s) (or their employer(s)) 2020. Re-use permitted under CC BY-NC. No commercial re-use. See rights and permissions. Published by BMJ.

For numbered affiliations see end of article.

Correspondence to Dr Francesco Peyronel; francesco.peyronel@gmail.com

\section{ABSTRACT}

Introduction In patients on maintenance haemodialysis (HD), intradialytic hypotension (IDH) is a clinical problem that nephrologists and dialysis nurses face daily in their clinical routine. Despite the technological advances in the field of $\mathrm{HD}$, the incidence of hypotensive events occurring during a standard dialytic treatment is still very high. Frequently recurring hypotensive episodes during HD sessions expose patients not only to severe immediate complications but also to a higher mortality risk in the medium term. Various strategies aimed at preventing IDH are currently available, but there is lack of conclusive data on more integrated approaches combining different interventions.

Methods and analysis This is a prospective, randomised, open-label, crossover trial (each subject will be used as his/her own control) that will be performed in two distinct phases, each of which is divided into several subphases. In the first phase, $27 \mathrm{HD}$ sessions for each patient will be used, and will be aimed at the validation of a new ultrafiltration (UF) profile, designed with an ascending/descending shape, and a standard dialysate sodium concentration. In the second phase, $33 \mathrm{HD}$ sessions for each patient will be used and will be aimed at evaluating the combination of different UF and sodium profiling strategies through individualised dialysate sodium concentration.

Ethics and dissemination The trial protocol has been reviewed and approved by the local Institutional Ethics Committee (Comitato Etico AVEN, prot. 43391 22.10.19). The results of the trial will be presented at local and international conferences and submitted for publication to a peerreviewed journal.

Trial registration number ClinicalTrials.gov Registry (NCT03949088).

\section{BACKGROUND}

\section{Introduction}

Removal of the interdialytic fluid gain by ultrafiltration (UF) is a mainstay of renal replacement therapy in patients with end-stage renal
Strengths and limitations of this study

- The trial has a prospective, randomised, open-label, crossover design; neither patients nor investigators can be blinded to the treatment assignment.

- Only hypotension-prone patients will be enrolled in the study, thus eliminating possible biases determined by the inclusion of haemodynamically stable patients.

- Several different strategies aimed at preventing intradialytic hypotension such as ultrafiltration profiling, sodium modelling, dialysate sodium individualisation and low dialysate temperature will be combined and tested at the same time.

disease. However, the time limitation intrinsic to the duration of a standard haemodialysis (HD) session may set the stage for haemodynamic instability. In fact, the patients with a large interdialytic weight gain (IDWG) and/or older patients with heart failure are especially prone to intradialytic hypotension (IDH). Hypotensive events occurring during a standard HD treatment are still very frequent, with a reported incidence varying between $5 \%$ and $30 \%$ depending on the definition of IDH. ${ }^{12}$ In view of the growing number of elderly patients with chronic kidney disease and a high cardiovascular comorbidity burden, who will likely need HD at some point in their clinical course, IDH is likely to remain a relevant clinical issue in the near future despite the technological advances in the field of HD.

IDH not only causes patient discomfort but may also contribute to severe consequences, such as delivery of an inadequate dialysis 
dose, vascular access thrombosis, as well as cardiac, cerebral and mesenteric ischaemia. ${ }^{3-6}$ Moreover, a strong linear correlation between IDH incidence and mortality has been described. ${ }^{78}$

In clinical practice, common interventions carried out in response to IDH include setting the patient in the Trendelenburg position, reducing or stopping the UF process and infusing normal saline to restore intravascular volume. In order to prevent IDH, various approaches have also been suggested based on the modulation of UF ('UF profiling'), qualitative changes in dialysate composition (eg, the use of high sodium concentrations) and lowering of dialysate temperature. Furthermore, in recent years, more sophisticated techniques such as blood volume (BV) monitoring and BV monitoring-based biofeedback systems have been developed aiming at the same goal. ${ }^{9-11}$ However, no conclusive data are currently available on more integrated approaches combining different interventions.

\section{UF-induced hypovolaemia: pathogenesis and compensatory mechanisms}

During a standard HD session, the aim of the UF process is to shift the patient from a state of hypervolaemia to a condition approaching 'dry weight', with usual relative decrease in total plasma volume by $10 \%-20 \% .{ }^{12}$ During plasma water removal with ensuing relative hypovolaemia, haemodynamic stability depends on body compensatory processes, the most important of which is plasma refilling. Compensatory refilling rates are usually lower than typical UF rates applied during HD. As a consequence, $\mathrm{BV}$ will gradually drop during treatment. ${ }^{9-12}$ In addition to plasma refilling, HD-induced hypovolaemia leads to the activation of cardiac and vascular compensatory mechanisms aimed at maintaining cardiac output and blood pressure (BP) values within the normal range.

\section{BV monitoring}

Several non-invasive methods to estimate relative BV (RBV) have been developed. These tools provide realtime and continuous assessment of plasma volume based on the modification of blood constituents (eg, haemoglobin) throughout the entire HD session. ${ }^{9} 13$

Several studies have investigated the relationships between RBV changes during HD, the trend of intradialytic BP values and the occurrence of IDH. ${ }^{14-19}$ However, most of these studies failed in demonstrating a link between the magnitude of RBV reduction and the occurrence of hypotensive episodes. RBV generally decreases in two distinct phases: (1) a rapid drop during the first hour of dialysis and (2) a slower decline in the following interval, suggesting an increased refilling of central veins due to BV shifting from the peripheral microcirculation towards the end of the treatment. ${ }^{20}$ Interestingly, systolic blood pressure (SBP) seems to follow the same downward trend, showing a rapid decrease in the first $25 \%$ of HD, independently of total UF volume or UF rate and a slower decline during the later phases, which is instead correlated to UF parameters. $^{21}$

The drop of RBV below an individual critical threshold is assumed to provoke intradialytic symptoms. However, RBV changes do not directly translate into absolute BV modifications, since these depend on the patient's current volume status. Indeed, the same per cent decrease in RBV may correspond to extremely different absolute volume changes, even in the same patient, and therefore it is impossible to identify a reliable limit for critical $\mathrm{RBV}$, which shows an interindividual variability ranging between $71 \%$ and $98 \%$ of the initial value. ${ }^{22}$

\section{UF profiling}

UF profiling is performed by variably shaping the UF process, instead of setting it at a constant rate, as is usually done in clinical practice (figure 1A). The UF rate shape can be set as a gradual or stepwise decrease (figure 1B,C), with higher rates at the beginning of the session, assuming that at this time point the patient is in a hypervolaemic state and can therefore better tolerate higher amounts of fluid removal during the first phase of treatment. In another possible UF profiling scheme (figure 1D), the UF process is interrupted intermittently, alternating phases in which the UF rate is set approximately to zero and other ones in which it is set to almost two times the value of conventional UF rates.

It has been suggested that stepwise and alternate UF profiling may be responsible for a greater number of symptomatic hypotensive episodes; conversely, the linear descending profile appears to be associated with fewer intradialytic adverse events. ${ }^{23}$

\section{Dialysate sodium}

Although sodium is clearly the most represented electrolyte in the dialysate, the optimal dialysate sodium concentration for patients undergoing chronic HD is still an unresolved issue. At present, in most dialysis centres, dialysate sodium is prescribed at a fixed, 'standard' concentration, without accounting for differences in plasma sodium levels among patients. Prescribed sodium concentration in the dialysate is typically higher than that in plasma, thus generating a positive gradient that causes sodium diffusion from dialysate to plasma. This leads to a positive sodium balance with ensuing increased thirst, greater IDWG and eventually volume-dependent hypertension. On the other hand, in the case of a negative concentration gradient, which develops when dialysate sodium concentration is lower than plasma levels, a disproportionate diffusive loss of the electrolyte may occur and may cause hypotensive events or cramps.

While a positive intradialytic sodium balance may be effective both in the prevention and as an acute treatment of intradialytic symptoms, it may also sustain a vicious cycle by hindering the achievement of patient's 'dry' weight and favouring the development of intradialytic complications during the following session. ${ }^{2425}$ 
A

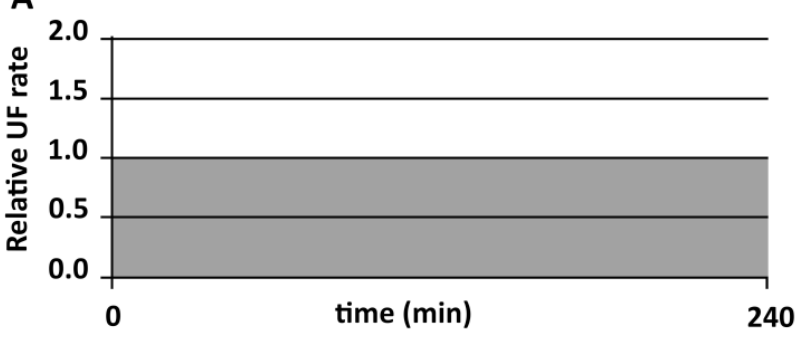

C

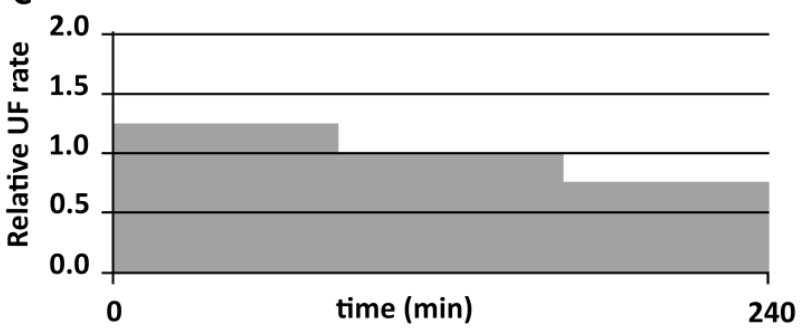

B

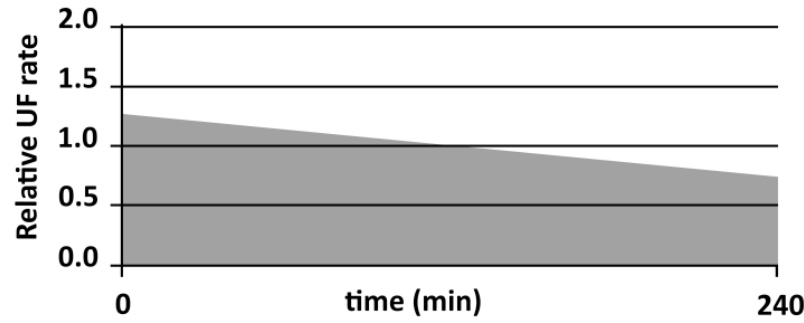

D

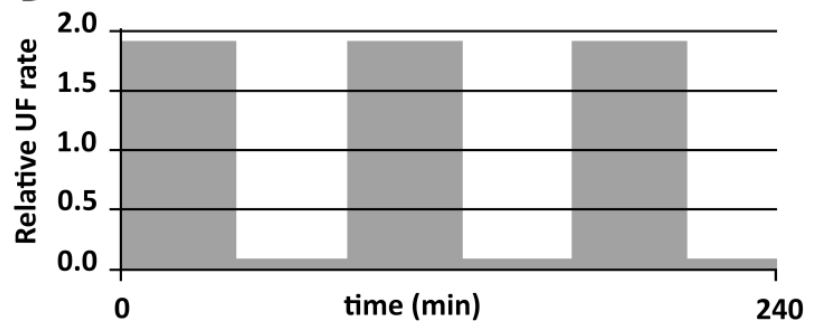

Figure 1 Ultrafiltration (UF) profiling during a 4-hour dialysis session. Constant linear UF rate (A). Linear descending UF profile (B). Stepwise descending UF profile (C). Alternate UF profile (D).

Predialytic serum sodium levels show a wide interindividual distribution, while intraindividual differences are negligible. ${ }^{25}$ According to the hypothesis of Keen and Gotch, each subject has an individual 'sodium setpoint', ${ }^{26}$ whereby an increase in serum sodium levels, as in the case of a diffusive influx from the dialysate, triggers thirst and increased fluid intake, so that serum sodium concentration can be brought back to the patient-specific setpoint. The increased interdialytic fluid intake leads to a greater IDWG, necessarily requiring higher UF rates and eventually predisposing the patient to a higher risk of IDH and cramps. Indeed, several studies have demonstrated that decreasing sodium balance through dietary restriction or the use of lower dialysate sodium concentrations may result in weaker thirst, reduced IDWG, lower BP values and improved echocardiographic parameters. ${ }^{27-33}$

An ideal treatment should remove the exact amount of sodium accumulated between two consecutive HD sessions, resulting in zero sodium balance. By adjusting net UF volume to match the total interdialytic fluid gain, it should be possible to obtain the removal of a quantity of sodium almost equal to that needed to achieve a neutral sodium balance. ${ }^{25}$ Consequently, in order to avoid diffusive sodium overload or depletion, it would be desirable to achieve a diffusive zero sodium gradient, which can be accomplished by aligning the dialysate sodium prescription to the patient's own serum sodium setpoint.

Conventional dialysis applies constant dialysate sodium levels throughout the entire dialysis session, whereas sodium profiling implies a dynamic modification of sodium levels along the treatment. Sodium profiling has been introduced in combination with UF profiling with the aim to obtain greater plasma osmolality, and thus refilling, in those treatment phases characterised by higher UF rates. Dialysate sodium can be lowered gradually or in a stepwise manner, with the latter method showing a stronger effectiveness in reducing intradialytic symptoms when compared with linear profiling. ${ }^{34} 35$ However, an inappropriate use of sodium profiling is one of the possible sources of dialysis-related sodium loading, causing increased IDWG and its complications. ${ }^{15} 252936$ The putative advantages associated with the use of sodium profiles with a neutral sodium balance need further investigation. However, a reduced number of intradialytic hypotensive events have been observed in some studies investigating the combination of neutral sodium balance profiles and UF profiling. ${ }^{36} 37$

\section{AIM OF THE STUDY}

The aim of the present study is twofold: (1) comparing different strategies of UF profiling, dialysate sodium individualisation/sodium profiling and their combination and (2) evaluating the effectiveness of a new UF profile which has been designed with an ascending/descending shape (figure 2).

The goal of the study is to provide better dialysis tolerance and lower rates of intradialytic hypotensive events by

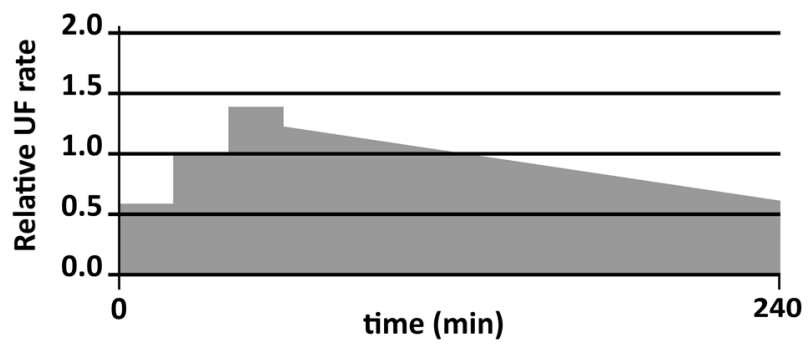

Figure 2 Ascending/descending ultrafiltration (UF) profile. 
the application of this UF profile design in combination with a neutral sodium balance.

\section{METHODS}

\section{Study design}

This is a prospective, randomised, open-label, crossover trial that will be carried out at a single centre. The study will be performed in two phases (see the Study phases section), each of which is divided in several subphases. Each subject will be used as his/her own control.

Participants will be enrolled among patients treated at the Dialysis Center of the Nephrology Unit of Parma University Hospital, Parma (Italy).

\section{Eligibility criteria-inclusion criteria}

- Written informed consent (consent form, informative sheet and confidentiality agreement are provided as online supplementary files 1-3).

- Age $\geq 18$ years.

- Three times weekly HD regimen for more than 6 months.

- 'Hypotension-prone patients': $\geq 2.1$ episodes in the nine sessions (ie, $\geq 3$ episodes of IDH in the month) preceding the run-in phase of the study, based on events reported in patients' charts.

\section{Eligibility criteria-exclusion criteria}

- IDWG $<1.4 \%$ of dry weight (corresponding to $<1 \mathrm{~kg}$ in a $70 \mathrm{~kg}$ person).

- Once or two times weekly HD regimen.

- Residual daily urine output $>300 \mathrm{~mL}$.

- Active acute disease or hospitalisation in the 8 weeks preceding the run-in phase.

\section{Study phases}

First phase: validation of the new UF profile with a standard dialysate sodium concentration. This phase will consist of 9 weeks of treatment (27 HD sessions) for each patient:

1. Run-in: constant Na concentration, constant UF rate2 weeks (six sessions).

2. Two-step descending Na profile, linear descending UF profile-3 weeks (nine sessions).

3. Washout: constant $\mathrm{Na}$ concentration, constant UF rate-1 week (three sessions).

4. Two-step descending Na profile, ascending/descending UF profile-3 weeks (nine sessions).

Patients will be randomly assigned to one of the following sequences:

(1), (2), (3), (4)

(1), (4), (3), (2).

Second phase: combination of UF profiles and testing of the contribution of an individualised dialysate sodium concentration. This phase will consist of 11 weeks of treatment (33 HD sessions) for each patient:

1. Run-in: standard constant Na concentration, constant UF rate-2 weeks (six sessions).
2. Individualised constant $\mathrm{Na}$ concentration, constant UF rate -2 weeks (six sessions).

3. Individualised two-step Na profile, linear descending UF profile-3 weeks (nine sessions).

4. Washout: individualised constant Na profile, constant UF rate-1 week (three sessions).

5. Individualised two-step $\mathrm{Na}$ profile, ascending/descending UF profile-3 weeks (nine sessions).

Patients will be randomly assigned to one of the following sequences:

(1), (2), (3), (4), (5)

(1), (2), (5), (4), (3).

Dry weight, antihypertensive medications and dialysis parameters will not be modified during the study phases, except for UF rate and dialysate sodium concentration. The patients who will be included in phase 1 will undergo a 2-week or longer washout period before entering phase 2. During these 2 weeks, dry weight and antihypertensive therapy may be re-evaluated and reassessed.

\section{Dialysis prescription}

Every patient will undergo a standard HD with the following prescription:

- Blood flow rate: individualised from $250 \mathrm{~mL} / \mathrm{min}$ to $350 \mathrm{~mL} / \mathrm{min}$ (this value will be established for each patient at the beginning of the run-in phase on the basis of previous evaluations and will not be changed for the whole duration of the study).

- Dialysate flow rate: $500 \mathrm{~mL} / \mathrm{min}$.

- Dialysate composition: $\mathrm{HCO}^{-} 34 \mathrm{mmol} / \mathrm{L}, \mathrm{K}^{+} 3$ $\mathrm{mmol} / \mathrm{L}, \mathrm{Ca}^{2+} 1.25 \mathrm{mmol} / \mathrm{L}, \mathrm{Mg}^{2+} 0.5 \mathrm{mmol} / \mathrm{L}, \mathrm{Cl}^{-}$ $111.5 \mathrm{mmol} / \mathrm{L}$, acetate $3.0 \mathrm{mmol} / \mathrm{L}$, glucose $1 \mathrm{~g} / \mathrm{L}$.

- Dialysate temperature: $36^{\circ} \mathrm{C}$ (we will allow the prescription of a dialysate temperature of $35.0^{\circ} \mathrm{C}$ or $35.5^{\circ} \mathrm{C}$, pending a possible patient discomfort as assessed by his/her reports of feeling cold. However, this issue has to be discussed with each patient and resolved before he/she has been enrolled in the trial; each patient will then undergo treatments with the same dialysate temperature for the entire duration of the study. Subsequent changes will not be permitted).

- Session duration: 4 hours.

During each dialysis, session patients will be allowed to drink a maximum amount of $150 \mathrm{~mL}$ of water, tea or coffee; eating a snack will also be allowed.

\section{UF profiles}

- 'Linear descending' UF profile: this profile provides a constantly decreasing UF rate during dialysis, starting at a UF rate 1.33 -fold the average UF rate $(33.25 \%$ of total UF rate).

- 'Ascending/descending' UF profile: this profile can be divided in two different phases. The first one includes three ascending steps during the first hour of treatment, with each step lasting 20 min (during the first step UF rate is set at $15 \%$ of total UF rate, during second step at $25 \%$ of total UF rate and during third step at $35 \%$ of total UF rate). During the following 
3 hours, UF rate is shaped as a linear descending UF profile, with a constantly decreasing UF rate, starting at a UF rate 1.33 -fold the average UF rate $(33.25 \%$ of total UF rate).

\section{Dialysate sodium}

First phase

- 'Standard' concentration: the investigators will deem as 'standard' a dialysate sodium concentration of $140 \mathrm{mmol} / \mathrm{L}$, which is the concentration usually prescribed in the local dialysis facility.

- Dialysate sodium profile will be shaped as a descending two-step ramp, with each step consisting of half the total treatment duration (2hours), and a $6 \mathrm{mmol} / \mathrm{L}$ difference between the concentrations set for each of the two steps. Assuming a one-compartment model with variable dialysate sodium, the profile will be set on the basis of an 'equivalent sodium'. This value will correspond to the dialysate sodium concentration expected to produce the same diffusive balance as that provided by a fixed standard concentration $(140 \mathrm{mmol} / \mathrm{L})$, that is, $144 \mathrm{mmol} / \mathrm{L}$ for the first 2 hours, $138 \mathrm{mmol} / \mathrm{L}$ for the last 2 hours.

\section{Second phase}

- 'Individualised' concentration: for each patient dialysate sodium concentration will be established on the basis of the average of the sodium plasma values measured by pre-HD sampling during the run-in phase (two repeated measurements before each HD session, for a total of 12 values for each patient). Plasma values will be measured by direct potentiometry.

- Dialysate sodium concentration will be set at the patient's average plasma sodium concentration.

- Dialysate sodium profile will be shaped as a descending two-step ramp, with each step consisting of half the total treatment duration (2 hours), with a $6 \mathrm{mmol} / \mathrm{L}$ difference between the concentrations set for each of the two steps. Assuming a one-compartment model with variable dialysate sodium, the profile will be set on the basis of an 'equivalent sodium'. This value will correspond to the dialysate sodium concentration expected to produce the same diffusive balance as that provided by a fixed individualised concentration (equal to the patient's average plasma sodium concentration), that is, 'average +4 ' $\mathrm{mmol} / \mathrm{L}$ for the first 2 hours, 'average -2 ' $\mathrm{mmol} / \mathrm{L}$ for the last 2 hours.

\section{Definition of 'dry weight', 'UF volume' and 'IDWG'}

- Dry weight will be estimated through standard clinical criteria.

- Total UF volume (net fluid to be removed) will be calculated before each session as the difference between patient's weight and his/her dry weight. A limit of $12.5 \mathrm{~mL} / \mathrm{kg} /$ hour will be considered as maximal total UF volume.

- IDWG will be calculated as the difference between patient's weight at the beginning of the dialysis session and the weight registered at the end of the previous session.

UF and IDWG will be corrected for pre-HD weight (UF\%) and dry weight (IDWG\%), respectively.

\section{Primary outcome and definition of 'IDH'}

The primary outcome will be the incidence of intradialytic hypotensive episodes. Hypotensive events and symptoms (headache, cramps, nausea and vomiting) will be recorded and analysed as both number of episodes and time of occurrence since the beginning of the HD session.

IDH will be defined as follows:

- 'Nadir90 IDH': minimum intradialytic SBP $<90 \mathrm{~mm}$ $\mathrm{Hg}$.

- 'Symptomatic IDH': decrease in SBP $\geq 20 \mathrm{~mm} \mathrm{Hg}$ or in mean arterial pressure (MAP) $\geq 10 \mathrm{~mm} \mathrm{Hg}$ associated with symptoms (Kidney Disease Improving Global Outcomes - KDIGO definition).

- 'Asymptomatic IDH': drop in BP ( $\mathrm{SBP} \geq 20 \mathrm{~mm} \mathrm{Hg}$ or MAP $\geq 10 \mathrm{~mm} \mathrm{Hg}$ ) within a $20 \mathrm{~min}$ interval (BP and heart rate will be recorded every $20 \mathrm{~min}$, regardless of symptoms).

- For patients whose SBP is $<100 \mathrm{~mm} \mathrm{Hg}$ at the beginning of treatment, the investigators will consider as IDH any decrease of SBP $\geq 10 \%$.

\section{Interventions in case of hypotensive events}

- Trendelenburg position.

- Temporary stop of UF ( $10 \mathrm{~min})$, then restart at a UF rate equal to 'total $\mathrm{UF}-100 \mathrm{~mL}$ '.

- Online infusion of $150 \mathrm{~mL}$ of saline solution.

- Discontinuation of the session.

\section{BV monitoring}

RBV will be evaluated through the BVM system integrated in the dialysis machine. $\mathrm{RBV}$ will be recorded every $10 \mathrm{~min}$.

\section{Secondary outcomes}

- Incidence of each component of the IDH definition.

- Pre-HD, intra-HD (after every hour of treatment) and post-HD plasma sodium levels as measured by direct potentiometry.

- BP and heart rate values recorded every $20 \mathrm{~min}$, or more frequently if clinically indicated, by machineintegrated Blood Pressure Monitoring (BPM).

- Achievement of UF, defined as:

- Achievement of dry weight: \%target $\mathrm{UF}_{\mathrm{DW}}=\mathrm{UF}$ vol/ (pre-HD weight-dry weight) $\times 100$.

- IDWG removal: \% target $\mathrm{UF}_{\mathrm{WG}}=\mathrm{UF}$ vol $/ \mathrm{IDWG} \times 100$.

- 'UF failure', defined as \% target $\mathrm{UF}_{\mathrm{DW}}<70 \%$.

- 'Session failure', defined as treatment discontinuation before $75 \%$ of the prescribed time (before 3 hours of treatment).

- Achieved spKt/V, as assessed by machine-integrated software (total body water calculated using Watson's equation). 


\section{Statistical analysis}

Sample size and assumptions

Based on our preliminary data, assuming an incidence of hypotensive events of 4/9 of HD sessions with a classic (linear) UF profile, and a reduction in the incidence of IDH by $2 / 9$ sessions with new (ascending/descending) UF profile, we estimated that at least 50 patients in a three-period three-treatment crossover trial would be needed to achieve $85 \%$ power to detect such difference between individualised ascending/descending UF profile and linear UF profile, with an alpha level of 0.05 , using a two-tailed test if the correlation between paired observations ranges between 0.1 and 0.5 . Therefore, we established a target sample of 60 patients, accounting for an approximate dropout rate of $20 \%$.

\section{Statistical methods}

We will analyse Bernoulli correlated balanced data employing multilevel mixed-effects logistic regression with unstructured covariance matrix, using the program melogit from Stata V.15.1 (2017 StataCorp, College Station, Texas, USA). Patients will be included as a random effect, whereas the indicator variable treatment, period and their interaction will be included as fixed effects. In secondary analyses, we will additionally fit population-average models adopting generalised estimating equations using the program xtgee from Stata V.15.1. Finally, we will estimate the difference in the mean systolic and diastolic BP (continuous variables) between UF profile treatment regimens using linear mixed-effects models with the program mixed from Stata V.15.1, in which patients will be included as a random effect, whereas treatment, period, dialysis session, hour of dialysis will be included as fixed effects.

\section{Patient and public involvement}

For this study protocol, there was no direct patient or public involvement.

\section{Ethics and dissemination}

The trial protocol has been reviewed and approved by the local Institutional Ethics Committee (Comitato Etico AVEN, prot. 43391 22.10.19). Participants will be provided with informative sheets describing in full detail trial aims, study phases, eligibility criteria, procedures/ interventions, data management, confidentiality and potential benefits/harms. They will be given the chance to discuss at any time any possible doubt with a member of the trial management committee. Prior to enrolment, an informed written consent will be obtained by one of the members of the trial management committee from all participants. Patients will be made aware that participation to this study is strictly voluntary and that consent can be withdrawn at any time. They will also receive a copy of the aforementioned documents.

Researchers will make every effort to preserve patients' confidentiality: alphanumeric codes for participants' identification will be assigned; data managers will store filled Case Report Forms (CRFs) containing patient's data in private locations with limited access; all databases will be password protected. All trial participants will be asked to sign a confidentiality form prior to enrolment.

Any additional healthcare need will be provided by the Italian National Health System; any potential harm derived by the participation to the trial will be covered by the investigating centre, that is, Parma University Hospital, Parma (Italy). Each study participant's follow-up after trial discontinuation will be provided by the Dialysis Center of the Nephrology Unit of Parma University Hospital, Parma (Italy).

The results of the trial will be presented at local and international scientific conferences and will be submitted for publication to a peer-reviewed journal. Data obtained by this study will be shared and available in deidentified form on reasonable request, wherever legally and ethically possible.

Any modification to the protocol impacting on the conduct of the study or the benefit of the participants will have to be communicated to and approved by the local Institutional Ethics Committee prior to the implementation.

\section{Current status of the trial}

The enrolment of patients has not begun yet and will commence in a few weeks. Patients' recruitment is expected to continue for at least 2 years.

\section{Author affiliations}

${ }^{1}$ Unità Operativa di Nefrologia, Azienda 0spedaliero-Universitaria di Parma, Parma, Emilia-Romagna, Italy

${ }^{2}$ Scuola di Specializzazione in Nefrologia, Università degli Studi di Parma Dipartimento di Medicina e Chirurgia, Parma, Emilia-Romagna, Italy

\section{Twitter Umberto Maggiore @Umaggiore}

Contributors FP: conception and design of the study, trial management, data collection and analysis, drafting of the article, critical revision of the article and final approval of the version to be published. EP: data collection, critical revision of the article and final approval of the version to be published. PF: improvement of the study design, critical revision of the article and final approval of the version to be published. GDB: improvement of the study design, critical revision of the article and final approval of the version to be published. GMR: data collection, critical revision of the article and final approval of the version to be published. UM: drafting of the article providing statistical expertise, data analysis, critical revision of the article and final approval of the version to be published. EF: improvement of the study design, trial management, drafting of the article, critical revision of the article and final approval of the version to be published. EF is the principal investigator. FP, EP, $\mathrm{PF}, \mathrm{GDB}$ and GMR are part of the trial management committee. Therefore, they will also be responsible for obtaining the signed informed consent from each participant and they will have to be available to solve any doubt that patients would present. FP, EP, GMR and other physicians involved in the protocol will be responsible for data collection. FP and UM will be responsible for data management, verification and analysis.For each treatment, the physician involved in patient's care and data collection will be responsible for discontinuing or modifying allocated interventions. The same person will collect, assess, report and manage adverse events (both solicited and spontaneously reported).

Funding The authors have not declared a specific grant for this research from any funding agency in the public, commercial or not-for-profit sectors.

Competing interests None declared.

Patient consent for publication Not required.

Provenance and peer review Not commissioned; externally peer reviewed. 
Open access This is an open access article distributed in accordance with the Creative Commons Attribution Non Commercial (CC BY-NC 4.0) license, which permits others to distribute, remix, adapt, build upon this work non-commercially, and license their derivative works on different terms, provided the original work is properly cited, appropriate credit is given, any changes made indicated, and the use is non-commercial. See: http://creativecommons.org/licenses/by-nc/4.0/.

ORCID iD

Francesco Peyronel http://orcid.org/0000-0001-8470-1952

\section{REFERENCES}

1 Chou JA, Kalantar-Zadeh K, Mathew AT. A brief review of intradialytic hypotension with a focus on survival. Semin Dial 2017;30:473-80.

2 Kuipers J, Verboom LM, Ipema KJR, et al. The prevalence of Intradialytic hypotension in patients on conventional hemodialysis: a systematic review with meta-analysis. Am J Nephrol 2019;49:497-506.

3 Chang TI, Paik J, Greene T, et al. Intradialytic hypotension and vascular access thrombosis. J Am Soc Nephrol 2011;22:1526-33.

4 Burton JO, Jefferies HJ, Selby NM, et al. Hemodialysis-Induced repetitive myocardial injury results in global and segmental reduction in systolic cardiac function. Clin J Am Soc Nephrol 2009;4:1925-31.

5 MacEwen C, Sutherland S, Daly J, et al. Relationship between hypotension and cerebral ischemia during hemodialysis. J Am Soc Nephrol 2017;28:2511-20.

6 Seong EY, Zheng Y, Winkelmayer WC, et al. The relationship between Intradialytic hypotension and hospitalized mesenteric ischemia: a case-control study. Clin J Am Soc Nephrol 2018;13:1517-25.

7 Flythe JE, Xue H, Lynch KE, et al. Association of mortality risk with various definitions of intradialytic hypotension. J Am Soc Nephrol 2015;26:724-34.

8 Chou JA, Streja E, Nguyen DV, et al. Intradialytic hypotension, blood pressure changes and mortality risk in incident hemodialysis patients. Nephrol Dial Transplant 2018;33:149-59.

9 Javed F, Savkin AV, Chan GSH, et al. Recent advances in the monitoring and control of haemodynamic variables during haemodialysis: a review. Physiol Meas 2012;33

10 Reeves PB, Mc Causland FR. Mechanisms, clinical implications, and treatment of Intradialytic hypotension. Clin J Am Soc Nephrol 2018;13:1297-303.

11 van der Sande FM, Dekker MJ, Leunissen KML, et al. Novel insights into the pathogenesis and prevention of Intradialytic hypotension. Blood Purif 2018;45:230-5.

12 Anand S, Sinha AD, Agarwal R. Determinants and short-term reproducibility of relative plasma volume slopes during hemodialysis. Clin J Am Soc Nephrol 2012;7:1996-2001.

13 Dasselaar JJ, Lub-de Hooge MN, Pruim J, et al. Relative blood volume changes underestimate total blood volume changes during hemodialysis. Clin J Am Soc Nephrol 2007;2:669-74.

14 Krepel HP, Nette RW, Akçahüseyin E, et al. Variability of relative blood volume during haemodialysis. Nephrol Dial Transplant 2000;15:673-9.

15 Oliver MJ, Edwards LJ, Churchill DN. Impact of sodium and ultrafiltration profiling on hemodialysis-related symptoms. J Am Soc Nephrol 2001;12:151-6.

16 Tonelli M, Astephen P, Andreou P, et al. Blood volume monitoring in intermittent hemodialysis for acute renal failure. Kidney Int 2002;62:1075-80.

17 Andrulli S, Colzani S, Mascia F, et al. The role of blood volume reduction in the genesis of intradialytic hypotension. Am J Kidney Dis 2002;40:1244-54.
18 Mitra S, Chamney P, Greenwood R, et al. Linear decay of relative blood volume during ultrafiltration predicts hemodynamic instability. Am J Kidney Dis 2002;40:556-65.

19 Booth J, Pinney J, Davenport A. Do changes in relative blood volume monitoring correlate to hemodialysis-associated hypotension? Nephron Clin Pract 2011;117:c179-83.

20 Javed F, Savkin AV, Chan GSH, et al. Assessing the blood volume and heart rate responses during haemodialysis in fluid overloaded patients using support vector regression. Physiol Meas 2009;30:1251-66.

21 Dinesh K, Kunaparaju S, Cape K, et al. A model of systolic blood pressure during the course of dialysis and clinical factors associated with various blood pressure behaviors. Am J Kidney Dis 2011;58:794-803.

22 Barth C, Boer W, Garzoni D, et al. Characteristics of hypotensionprone haemodialysis patients: is there a critical relative blood volume? Nephrol Dial Transplant 2003;18:1353-60.

23 Donauer J, Kölblin D, Bek M, et al. Ultrafiltration profiling and measurement of relative blood volume as strategies to reduce hemodialysis-related side effects. Am J Kidney Dis 2000;36:115-23.

24 Locatelli F, Covic A, Chazot C, et al. Optimal composition of the dialysate, with emphasis on its influence on blood pressure. Nephrol Dial Transplant 2004;19:785-96.

25 Thijssen S, Raimann JG, Usvyat LA, et al. The evils of intradialytic sodium loading. Contrib Nephrol 2011;171:84-91.

26 Keen ML, Gotch FA. The association of the sodium "setpoint" to interdialytic weight gain and blood pressure in hemodialysis patients. Int J Artif Organs 2007;30:971-9.

27 de Paula FM, Peixoto AJ, Pinto LV, et al. Clinical consequences of an individualized dialysate sodium prescription in hemodialysis patients Kidney Int 2004;66:1232-8.

28 Kayikcioglu M, Tumuklu M, Ozkahya M, et al. The benefit of salt restriction in the treatment of end-stage renal disease by haemodialysis. Nephrol Dial Transplant 2009;24:956-62.

29 Hecking M, Kainz A, Hörl WH, et al. Sodium setpoint and sodium gradient: influence on plasma sodium change and weight gain. Am J Nephrol 2011;33:39-48.

30 Munoz Mendoza J, Bayes LY, Sun S, et al. Effect of lowering dialysate sodium concentration on interdialytic weight gain and blood pressure in patients undergoing thrice-weekly in-center nocturnal hemodialysis: a quality improvement study. Am J Kidney Dis 2011;58:956-63.

31 Arramreddy R, Sun SJ, Munoz Mendoza J, et al. Individualized reduction in dialysate sodium in conventional in-center hemodialysis. Hemodial Int 2012;16:473-80.

32 Zhou Y-L, Liu J, Ma L-J, et al. Effects of increasing diffusive sodium removal on blood pressure control in hemodialysis patients with optimal dry weight. Blood Purif 2013;35:209-15.

33 Wong MMY, McCullough KP, Bieber BA, et al. Interdialytic weight gain: trends, predictors, and associated outcomes in the International dialysis outcomes and practice patterns study (DOPPS) Am J Kidney Dis 2017;69:367-79.

34 Meira FS, Figueiredo AE, Zemiarcki J, et al. Two variable sodium profiles and adverse effects during hemodialysis: a randomized crossover study. Ther Apher Dial 2010;14:328-33.

35 Dunne N. A meta-analysis of sodium profiling techniques and the impact on intradialytic hypotension. Hemodial Int 2017;21:312-22.

36 Song JH, Park GH, Lee SY, et al. Effect of sodium balance and the combination of ultrafiltration profile during sodium profiling hemodialysis on the maintenance of the quality of dialysis and sodium and fluid balances. J Am Soc Nephrol 2005;16:237-46.

37 Zhou YL, Liu HL, Duan XF, et al. Impact of sodium and ultrafiltration profiling on haemodialysis-related hypotension. Nephrol Dial Transplant 2006;21:3231-7. 